\title{
DISCOVERING ARABIC RHYTHM THROUGH A SPEECH CYCLING TASK*
}

\author{
Bushra Adnan Zawaydeh", ${ }^{*,}$, Keiichi Tajima ${ }^{+, \dagger}$ and Mafuyu Kitahara \\ ${ }^{*}$ Lernout \& Hauspie Speech Products \\ ${ }^{\star}$ ATR International (Kyoto, Japan) \\ †Indiana University
}

\section{Introduction to the Problem}

\subsection{Background}

In traditional descriptions of speech rhythm, there is a well-known dichotomy about how languages vary in rhythmic structure. Languages have been classified as "stress-timed" (e.g., English, Arabic, and Russian), and "syllable-timed" (e.g., French and Spanish) (Pike, 1945; Jones, 1948; Abercrombie, 1967). This classification was based on an impressionistic description that identifies which units (stressed syllables or individual syllables) tend to occur isochronously, or at regular time intervals. A third type of languages-"mora-timed" languages - has also been proposed (Han, 1962; Hoequist, 1983). One of the best examples of a mora-timed language is Japanese in which moras occur at equal intervals. Hence, bimoraic heavy syllables are twice as long as monomoraic (or light) syllables.

Two main questions are addressed in this paper. (1) If we examine the temporal organization of languages across the traditional

\footnotetext{
${ }^{*}$ We thank Professors Robert Port and Kenneth de Jong for their exquisite comments. We also thank the audiences of the 1998 Mid-Continental Workshop on Phonology (Ann-Arbor, Oct 18, 1998) and the $13^{\text {th }}$ Annual Symposium on Arabic Linguistics (Stanford, Mar 5, 1999) for their questions and comments.
} 
rhythmic categories, will we find measurable differences between stress-timed languages and mora-timed languages? (2) Moreover, if we examine languages within a single rhythmic category, are they really the same in their rhythmic structure?

With regards to experimental studies on rhythm in Arabic, Miller (1984: 75) investigated the degree of consensus on the perception of stress/syllable-timed rhythm in seven languages. Recorded samples of these languages were presented to French and English phoneticians and non-phoneticians. The results showed that Arabic was classified by these non-speakers of Arabic as a "strongly stress-timed" language.

However, in experimental studies of Arabic rhythm, phonetic measurements failed to find strict isochrony. Roach (1982) studied a group of languages that were characterized by Abercrombie to be syllable-timed (French, Telegu and Yoruba) and stress-timed (English, Russian, and Arabic). He was not successful in finding measurements of time intervals that could classify these languages into two separate groups. Hence, Roach concluded that "the basis for the distinction is auditory and subjective. A language is syllable-timed if it sounds syllable timed".

Heliel (1982: 91) studied the rhythm of modern literary Arabic, by recording his own voice while reading a short Arabic passage. Heliel divided the passage into rhythmic units. He defined a rhythmic unit as being one that "starts with a stress and contains everything that follows that stress up to, but not including the next stress". Measurements of these rhythmic units began at the onset of the stressed syllable. Heliel's goal was to find if there is any evidence for a tendency for isochrony in Arabic. He found that "interstress times which correspond to rhythmic units, vary in duration and the variation is great" (p. 100). The duration of the rhythmic unit depended on how many syllables it contains and the type of syllables that are involved.

Tajima and Port (to appear) have also reported that in both English and Japanese, acoustic studies that measured the duration between stressed syllables or the duration of individual moras either could not find isochrony in these languages or only found a tendency toward it. Hence, phonetic studies that used duration measurements have not been able to find evidence that supports typological labels that linguists proposed, such as stress timing and syllable timing. (Shen \& Peterson, 1982; Bolinger, 1965; O'Connor, 1965; Uldall, 1971; Williams \& Hiller, 1994). 


\subsection{Speech cycling}

Recently, a novel experimental method has been developed to study speech rhythm and its variation across languages (Cummins \& Port 1998; Port, Tajima \& Cummins 1998; Cummins 1997; Tajima 1998). In this general class of tasks, called speech cycling, speakers produce a phrase repeatedly in time with a periodic auditory stimulus, such as a metronome. Tajima and Port (to appear) explain that using such a task encourages the participant to produce highly rhythmic forms of speech, since the speaker aligns some part of the sentence with the metronome. Speech produced in this task resembles rhythmic speaking styles that one might encounter in commonplace settings, such as singing, cheering in sporting events, chanting, list reading and so on. Also, repeated speech behavior is also witnessed as early in human development as the reduplicative babbling of prelinguistic infants (Stark, 1986).

As Tajima (1998: 100) further points out, repetition tasks are not unique to speech cycling tasks. Repetition of speech materials has been used in studies of syllable organization (Stetson, 1951; de Jong, 1999), as well as studies on so-called "P-center" (perceptual moment of occurrence) effects (Morton et al, 1976; Marcus, 1981; Scott, 1993). Hence, it seems reasonable to adopt repetitive forms of speech in studying whether the difference between stress-timed and syllabletimed languages might be detected acoustically.

The speech cycling task has been successful in revealing rhythmic characteristics of English speech. Cummins (1997) instructed native English speakers to repeat short phrases such as "beg for a dime". When subjects repeat this phrase, each repetition of the phrase could be considered a cycle; hence the first "beg" to the second "beg" would constitute a complete cycle. A relative measure of timing, called phase angle, can then be used to express the time of occurrence of the word "dime" relative to this cycle (see Section 2.4 for a precise definition of phase). Cummins found that when subjects were asked to align the beginning of the phrase (beg) along with the metronome beat, the syllable "dime" occurred mostly either at one-third, one-half, or two-thirds of the way in the cycle from "beg" to "beg". Timing patterns other than these three were difficult to reproduce when the subjects were given metronome signals to locate the stressed syllable "dime" at various arbitrary phase angles. Thus, in English the stressed syllables in the speech cycling task were found to occur at 
phases $1 / 3,1 / 2$, and $2 / 3$, i.e., values that can be expressed as simple fractions. We refer to these as "simple harmonic phases".

Tajima (1998) used the speech cycling task to compare the rhythmic structures of Japanese and English. He found that when the metronome period is increased gradually across trials, speakers fall in and out of stable, quasi-discrete rhythmic modes. This was found among both English and Japanese speakers.

English and Japanese were found to differ, however, in the types of syllables that were produced near simple harmonic phases (Tajima, 1998). While English speakers tend to place the last stressed syllable (e.g., "de-" in "great Bombay demons") halfway between the start of two successive repetitions, Japanese speakers tend to place the phrase final syllable (e.g., "da" in "doku dami da") halfway between the start of two successive repetitions. Tajima proposed that these languages behaved in different ways because they differ in what they consider to be prominent linguistically in the phrase. In Japanese, it is the last syllable that is prominent, while in English it is the last stressed syllable that is prominent.

Through additional experiments, Tajima (1998) further tested whether English and Japanese behave differently in speech cycling tasks, by examining the isochrony of stressed syllables in English vs. accented syllables in Japanese. Tajima hypothesized that these two types of syllables may potentially behave similarly in speech cycling tasks because they are similar in their effects on fundamental frequency patterns (Beckman, 1986), and they are both considered prominent in metrical theory (Haraguchi, 1991). The traditional rhythmic labels, however, make different predictions. That is, if English is "stress timed" then stressed syllables should show a tendency towards isochrony. Addition of extra syllables in the phrase should then be resolved by a resistance in the temporal displacement of the stressed syllables. In comparison, if Japanese is a "mora-timed" language, then addition of extra syllables in the phrase should result in the displacement of the accented syllable; these syllables should show no tendency to occur at regular intervals. Therefore, the prediction is that Japanese accented syllables should show more temporal displacement than English stressed syllables.

Tajima conducted a speech cycling experiment and examined the effect of the addition of an extra syllable on the timing of phrases in Japanese and English. He found that in Japanese, the addition of a syllable before the accented syllable results in the displacement of the 
beat of the accented syllable to the right. The addition of a syllable after the accented syllable results in the displacement of the accented syllable to the left. A comparable experiment was done on English. It was found that the temporal displacement resulting from the addition of an extra syllable was much smaller in English than in Japanese. This suggests that English stressed syllables more closely approximate isochrony than do Japanese accented syllables.

In short, the speech cycling method has been successful in revealing physical correlates of rhythmic variation between English and Japanese. In this paper, we examine the temporal organization of another language, Arabic, which has been labeled by linguists as being "stress timed". We will explore the possibility of finding measurable differences between the stress timed languages, English and Arabic, and the mora timed language, Japanese. We will also try to find out if there is any category-internal differences in stress timed languages like English and Arabic.

In the following sections, we will report on an experiment that we conducted to examine the rhythmic structure of Arabic. We will compare the temporal organization of this language to those of English and Japanese, which have been studied by Tajima (1998).

\section{Method}

\subsection{Subjects}

The subjects of this study were two male and two female native speakers of Ammani-Jordanian Arabic, between 16 and 28 years of age. All speakers were fluent in English, and have knowledge of French. However, the only language that was used at the time of the recording was Arabic.

\subsection{Stimuli}

Sets of 50-ms, $600-\mathrm{Hz}$ pure tones were generated on a workstation and recorded on an analog tape. These pure-tone beeps were recorded at fifteen different metronome periods which we call "trials". The slowest metronome period was $1500 \mathrm{msec}$, and the fastest was 554 msec. The metronome period decreased by $7 \%$ on successive trials, resulting in a continuum of decreasing metronome periods across fifteen trials. Each trial consisted of 12 beeps with a fixed period. Beeps were presented to subjects through headphones. 


\subsection{Procedure}

Subjects spoke into a microphone, and their voice was recorded on a tape using a Marantz professional portable tape recorder. The recording took place in a quiet room in Amman, Jordan. The subjects were instructed to listen to the first four metronome beeps and then to start repeating the phrase once per beep, aligning the beginning of the phrase with each beep. After eight repetitions, they were cued to stop repeating the phrase. They were instructed not to insert breaths between repetitions, since previous experience suggests that breathing disrupts the rhythmic pattern.

Subjects started with the slowest trial, then performed successively faster trials. When the metronome period became too fast, they moved on to the next phrase, starting again from the slowest trial. The shortest period one of the subjects was able to achieve was about 650 msec for a 4-syllable phrase. On average, each subject repeated a given phrase at about 10-12 different rates.

\subsection{Materials}

The materials used are given in (1). We indicate whether the syllables in each word are stressed $(\mathrm{S})$ or unstressed (u). Each phrase contains three stressed syllables which were the target syllables for later measurements. The first syllable in all phrases is stressed, but the phrases differ in whether the third stressed syllable occurs phrasefinally-(1a), (1c), and (1e)—or in penultimate position-(1b), (1d), and (1f). The phrases also differ in the number of unstressed syllables that occurred between the first and second stressed syllables. Phrases (1a) and (1b) have no intervening syllable between the first and second stressed syllables. Phrases (1c) and (1d) have one intervening unstressed syllable, and phrases (1e) and (1f) have two. The number of unstressed syllables between the second and third stressed syllables was always one.

\# syllables Gloss

$4 \quad$ "Baasim's wife is May."
$5 \quad$ "Naadir's daughter is Diima."
$5 \quad$ "Badir built a house."
$6 \quad$ "Tomorrow, Baasil will regret it."


e. $\mathrm{S}$ u u $\mathrm{S}$ u $\mathrm{S}$ bulbul binnan badiin

f. $S$ u u $S$ u $S$ u basma $_{i}$ badaal madiine
6 "Binnan's nightingale is fat."

$7 \quad$ "I listen for a city."

Of the eight repetitions of a phrase in each trial, measurement was taken from five repetitions. The first two and the last repetitions were discarded because subjects were often less stable at the start and end of a trial than in the middle.

\subsection{Phase}

We follow an approach which was found to be fruitful in several earlier studies conducted by Cummins, Port, and Tajima. This method involves taking the relative timing of beats instead of absolute measures, such as milliseconds. The relative measure can be defined in two ways by taking two different baselines. The two measures are referred to as external phase and internal phase.

To illustrate what we mean by external phase and internal phase, we can use as an example the repetition of the phrase "mart baasim may". As illustrated in Figure 1, external phase takes as the baseline the interval between the initial beats of the current and the following repetitions, and measures the time of occurrence of a syllable-e.g., the final stressed syllable "may"-relative to this baseline (i.e., b/a). On the other hand, internal phase takes as the baseline the interval between the initial and final stressed syllable beats of a single repetition, and measures the timing of the medial stressed syllable beat relative to this baseline (i.e., $c / b$ ).

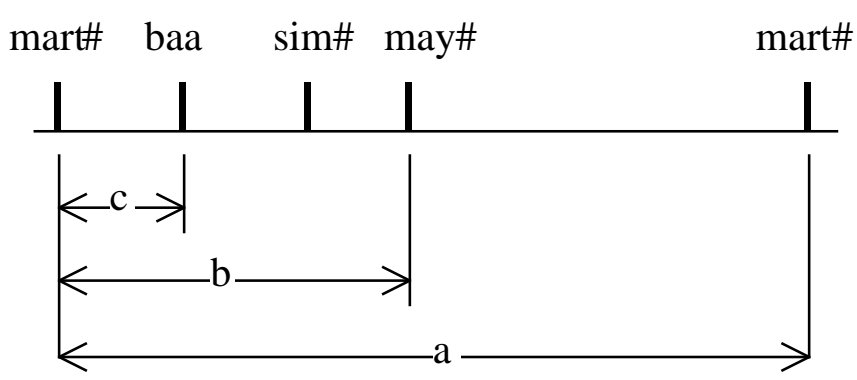

Figure 1: Definition of phases. Internal phase $=\mathrm{c} / \mathrm{b}$. External phase $=\mathrm{b} / \mathrm{a}$. 
Tajima (1998) explains that there are three reasons that motivate the use of phase as a metric, as opposed to using absolute duration. First, by using phase, one can measure time by referring to the periodicity of what is being measured. Hence, there is no need to refer to the arbitrary scale of milliseconds. Second, using phase is appropriate because rhythm is relational. Two successive time intervals could, for instance, be related to each other in a 2-to-1 ratio (2:1). Absolute measures can capture 1:1 ratios as equal, isochronous intervals, but it cannot account for 2:1 ratios in a simple manner. By using phase, however, the 2:1 ratio can be expressed as a simple harmonic fraction, such as two-thirds. Third, phase can normalize tempo differences. Hence, rhythmic regularities could be examined and compared across different metronome rates.

\section{Results}

We first examine the results in terms of both absolute time and external phase. Figure 2 shows sample data from one block of trials for the Arabic phrase "mart baasim may" produced by one speaker. Fourteen different metronome rates are shown on the y-axis, with the slowest trial at the top and the fastest at the bottom. Time in milliseconds is plotted on the x-axis. Each tick mark shows the timing of each syllable beat across five repetitions in each trial. As the metronome gets faster, the initial beat of the next repetition comes closer to the end of the current repetition. As the speech rate is increased gradually, the interval between the last syllable of a repetition ("may") and the initial syllable of the next repetition ("mart") becomes shorter while other intervals are relatively intact in duration.

In Figure 3, we present the same data as in Figure 2, but now in terms of mean external phase. Taking the external phase involves stretching the time axis of trials in faster rates and aligning the rightmost beats of those trials with that of the slowest trial. Doing this results in normalizing the tempo. 


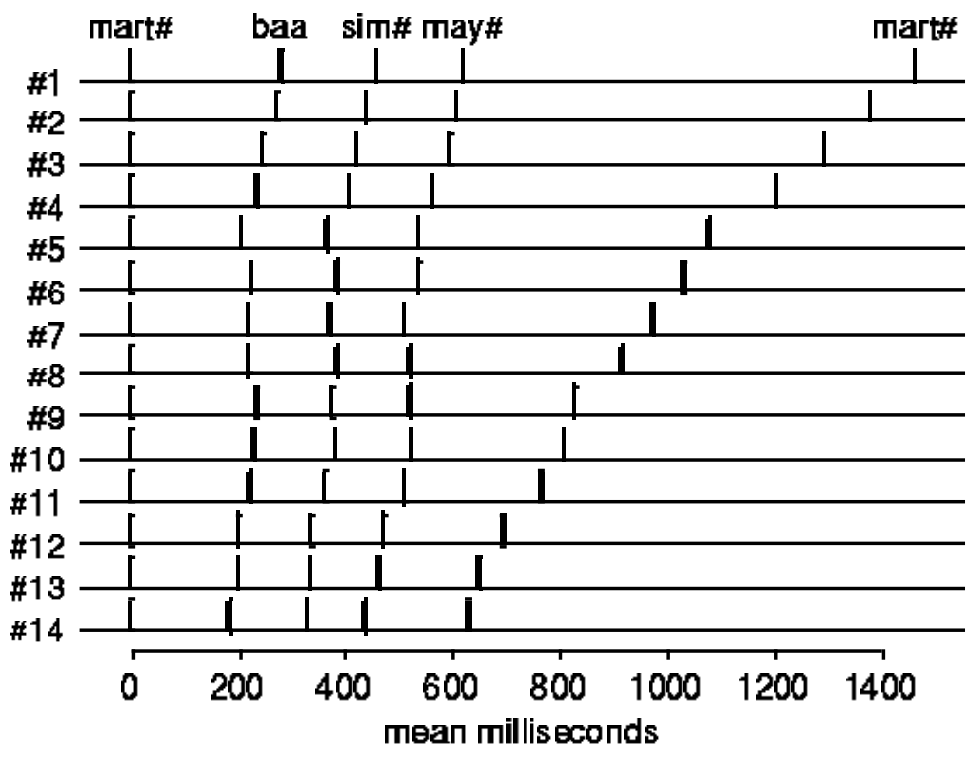

Figure 2: Sample measurements of syllable beats in absolute time.

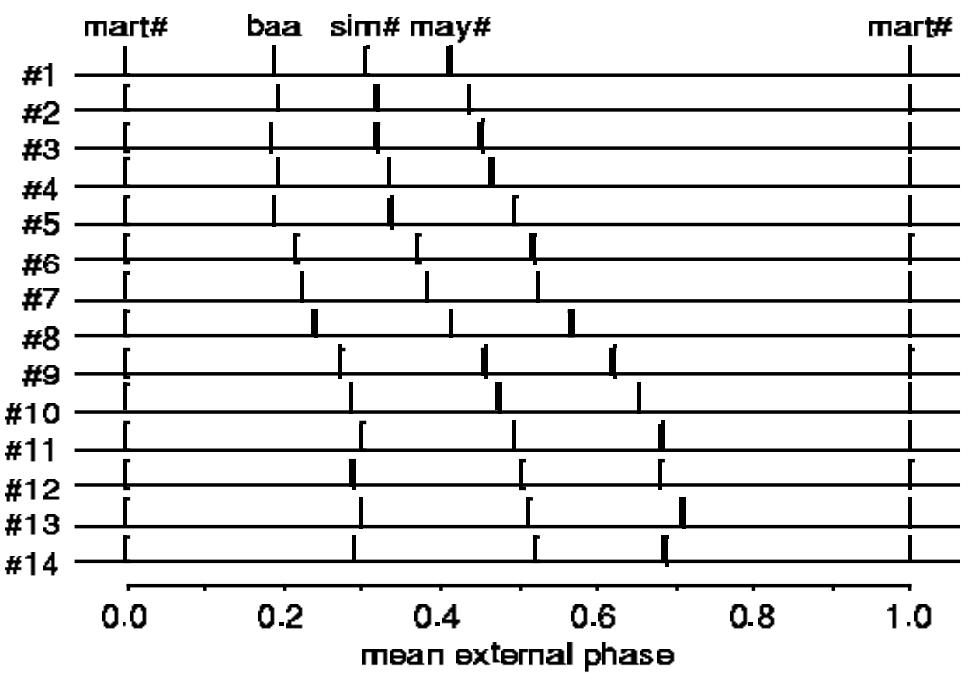

Figure 3: Sample measurements of syllable beats in external phase. 


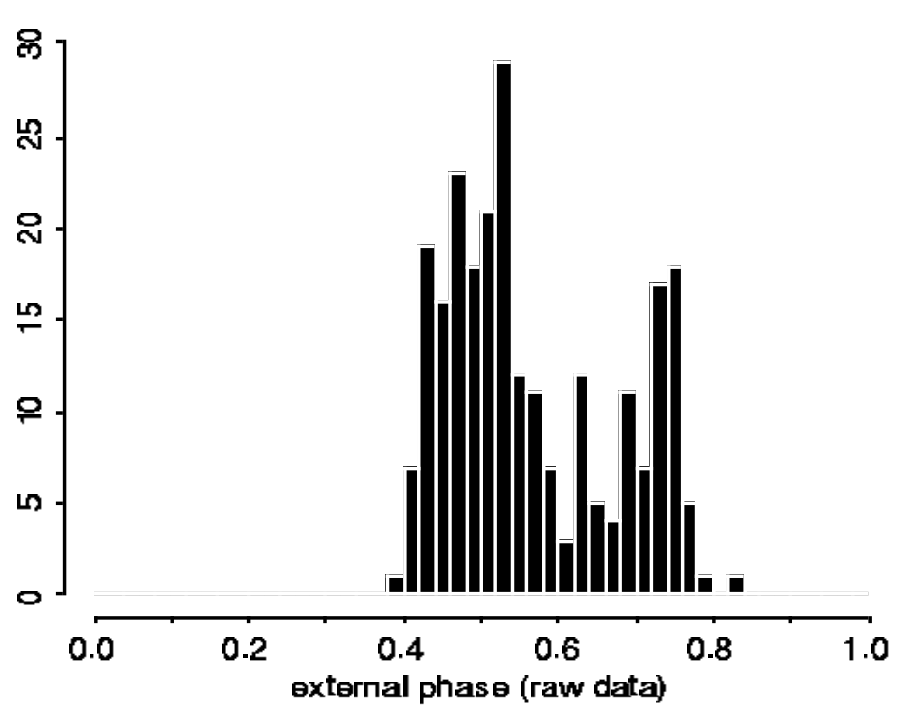

Figure 4: Sample histogram of the beat of the last syllable in external phase.

A crucial observation to note is that even though the metronome period decreases steadily ( $93 \%$ of the previous), the external phase of the final syllable falls in and out of two preferred regions. As metronome period decreases from top to bottom in Figure 3, the final syllable shows a relatively monotonic increase in external phase. However, the rate of change in external phase is smaller in magnitude among some trials than others. We interpret the relatively smaller changes in external phase as indicating a quasi-discrete, stable rhythmic mode. The first mode is demonstrated by the first seven trials. Observe that the final beat is located at about 0.5. Further observe that in trials, 8,9 , and 10 , the final syllable "may" has fallen out of the stable rhythmic mode. Finally, in trials 11-14, a second mode occurs at about external phase 0.7 .

These modes of the final syllable beat become more evident by creating a histogram of observed external phases. In Figure 4, the data are collapsed across all trials and across all four Arabic speakers. Individual repetitions are shown instead of trial means. The most prominent mode is represented by the peak located around 0.5. The second prominent mode is found between 0.7 and 0.8 . 


\subsection{External phases in Arabic, English, and Japanese}

In order to understand the Arabic results, we will compare them to English and Japanese. The data that we will present for English and Japanese are from Tajima (1998), and were gathered by the same method that was used for gathering the Arabic data, using comparable text materials. The phrases in both languages were 4-8 syllables long, given in (2) and (3). The participants were four American English speakers (from Illinois, Indiana, North Carolina, and Texas) and four speakers of the Eastern dialect of Japanese (two from Fukushima, one each from Chiba and Nagano). All subjects were students at Indiana University.

The four English phrases in (2) differ in whether or not there is a final unstressed syllable, and also whether or not there are two medial unstressed syllables between the first and second stressed syllables. Syllables with primary lexical stress are marked "S", and all other syllables, including stressless and secondary stress syllables, are marked "u".

The four Japanese phrases presented in (3) differ in the number of syllables. Hence, (2a) has 5 syllables, while (2d) has 8 syllables. Each syllable is indicated with "s". All Japanese phrases were composed of light syllables which are equivalent to moras. Heavy syllables with two moras were not included in our corpus; as such "moras" and "syllables" are interchangeable terms for the Japanese corpus. The corpora were designed to focus on the difference not between syllable-timed and mora-timed languages, but rather between stresstimed and non-stress-timed languages.

(2) English Phrases
$S$ S u $S$
a. buy\# Doug\# a\# beer
b. great\# Bombay\# demons
$\mathrm{S} \mathrm{u}$ u $\mathrm{S}$ u $\mathrm{S}$
c. Betty\# fogot\# the\# bag

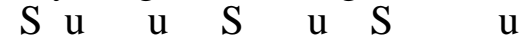
d. giving\# the\# girl\# a\# dough\# nut

\footnotetext{
${ }^{1}$ For the word "Bombay", lexical stress normally falls on "-bay". However, because the following word "demons" begins with a stressed syllable, it is in a clashing context, and the English Rhythm Rule (Hogg and McCully, 1987) alters the stress pattern to "BOMbay". Informal judgments suggested that the four English speakers in the study used this stress pattern.
} 
(3) Japanese Phrases
$\mathrm{S} S \mathrm{~S} \quad \mathrm{~S} \mathrm{~S}$
a. doku\# dami\# da
S S S S S S
b. dono\# garasu\# do
$\begin{array}{lllllll}S & S & S & S & S & S & S\end{array}$
c. goma\# dare\# dake\# da S S S S S S S S
d. doku\# dami\# darake\# da
It's a doku-dami plant.
Which glass door?
It's only the sesame sauce.
It's full of doku-dami plants.

First, we will present the Japanese results. Then we will compare the Japanese rhythmic system with the Arabic one. Finally, we will introduce the English results. We will see that when Japanese subjects do the cycling task, they place the last syllable of the phrase near phase 0.5. In comparison, in Arabic and English, it is not the last syllable that is placed near 0.5 , but rather the last stressed syllable.

\subsubsection{External phase in Japanese}

In this section we will take a brief look at Tajima's (1998) external phase data in Japanese. As mentioned earlier, external phase focuses on where the last syllable in the phrase occurs in relation to the first syllable of the phrase and the first syllable in the immediately following repetition of the phrase. Hence, in a phrase such as "doku dami da", we are targeting the last syllable "da", and we are interested in knowing where that syllable occurs in relation to the first syllable "do" in the current repetition and "do" in the following repetition.

In Figure 5, the last syllable beat of the phrase (shown with thick lines) occurs close to 0.5 . Hence, the Japanese speakers prefer a rhythmic mode which would align the final syllable beat near external phase 0.5 . 


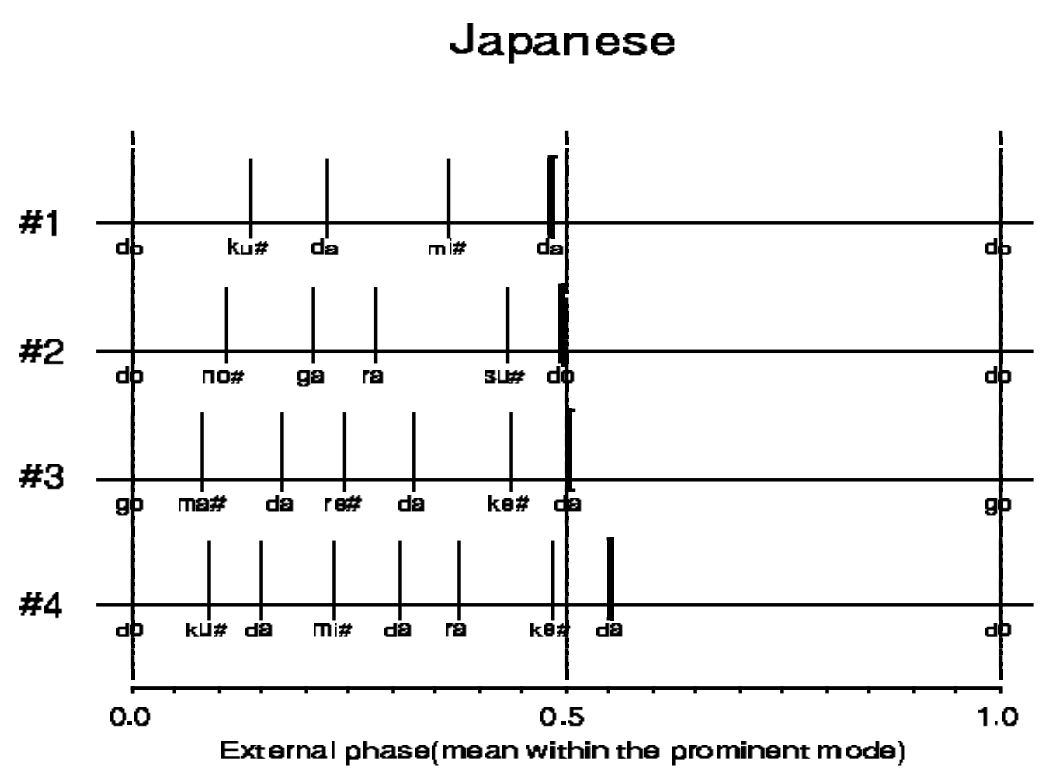

Figure 5: External phase data in Japanese (Tajima 1998).

\subsubsection{External phase in Arabic}

Figure 6 illustrates the external phase data in Arabic. Looking at the last syllables of the six phrases, unlike Japanese, they vary in where they occur within the cycle from one repetition onset to the next. There are some phrases, such as the $2 \mathrm{nd}$, 4th, and 6th, in which the last syllable occurs at about 0.7 .

However, looking at the timing of the last stressed syllable, we observe that it occurs at approximately phase 0.5 across all the six phrases. In all rhythmic modes preferred by the English speakers, the final stressed syllable shows a close alignment with the vertical line at 0.5 . In the next section, we will compare the Arabic and Japanese results with the English results. 


\section{Arabic}

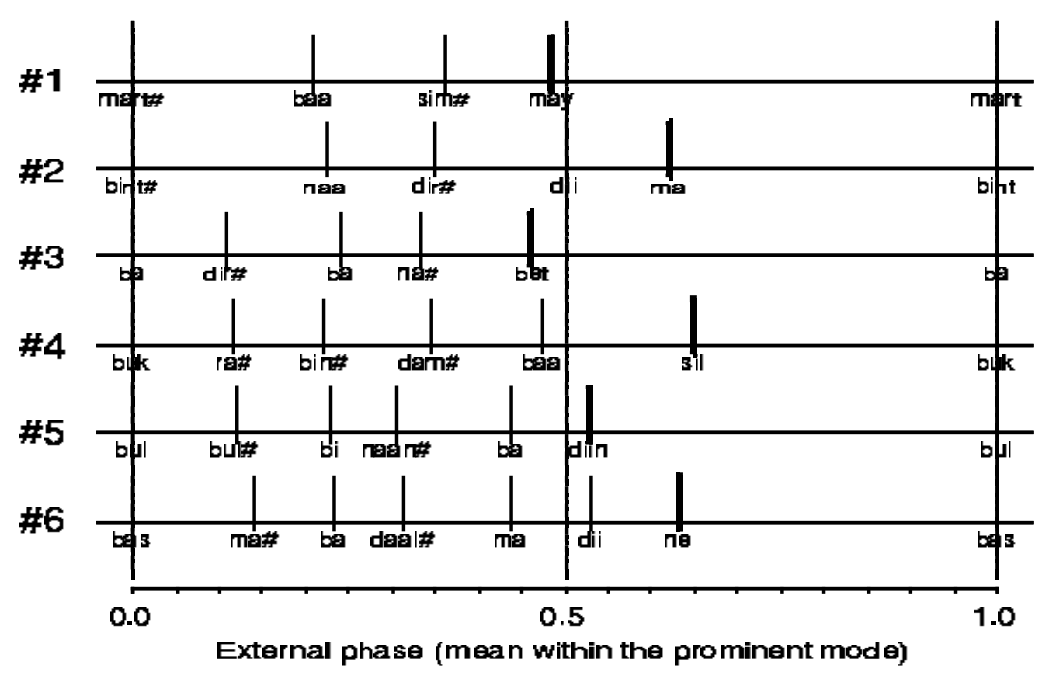

Figure 6: External phase data in Arabic.

\subsubsection{External phase in English}

Figure 7 presents the external phase data for English, taken from Tajima (1998). The figure shows that it is not the final syllable that is aligned with the simple harmonic phase at 0.5 . The last syllable in two phrases ("great Bombay demons" and "giving the girl a dough-nut") do not occur at 0.5. They occur much later, at about 0.7. However, if we observe the final stressed syllables in the four phrases, they occur at about 0.5 in all cases. Hence, English is similar to Arabic in that it is the last stressed syllable that occurs near 0.5. In comparison, Japanese is quite different in that it is the last syllable in the phrase that occurs at about 0.5 . In the next section we will take a closer look at English and Arabic by considering the internal phase measurements, in which the focus will be on how the medial stressed syllable of the phase is aligned in comparison with the first and final stressed syllables. 


\section{English}

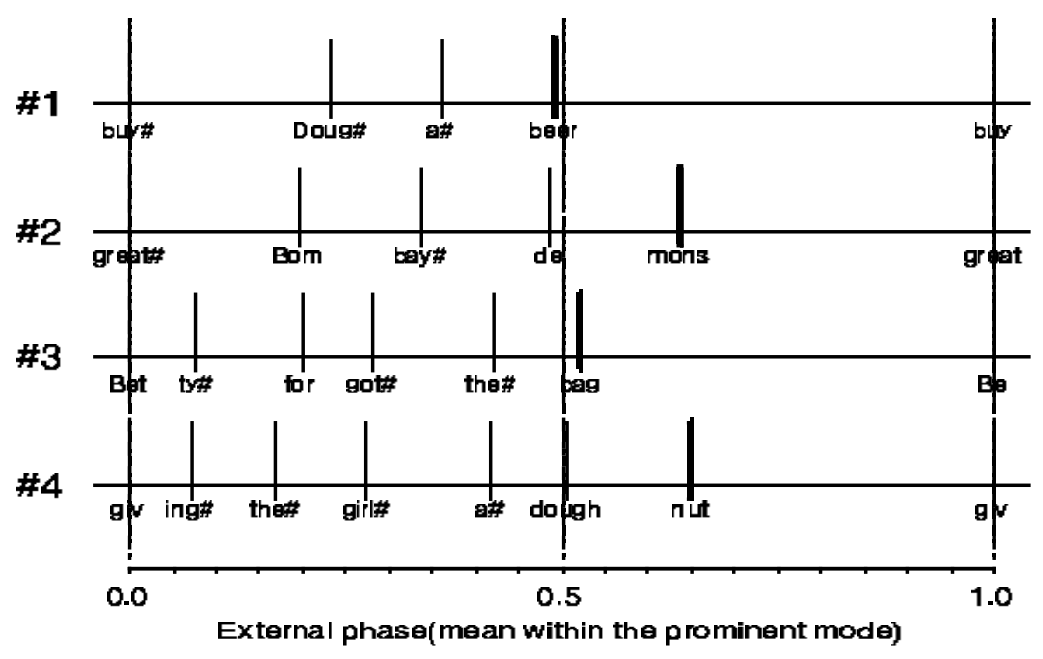

Figure 7: External phase data in English (Tajima 1998).

\subsection{Internal phase in Arabic and English}

In this section we will compare the results of the internal phase measurement for Arabic and English. Among the Arabic and English phrases shown in (1) and (2), a subset of them which matched in stress pattern and number of syllables are chosen for analysis, shown in (4). The stressed syllables are in bold.

(4) \# syllables Arabic
a. 4
b. 5
c. 6
d. 7
bint naadir diima
bulbul binaan badiin
basma $_{i}$ badaal madiine

English

buy Doug a beer

great Bombay demons

Betty forgot thebag

giving the girl a doughnut

Arabic and English phrases in each row of (4) match in stress pattern, schematically shown in the top part of Figure 8. For example, each phrase in (4a) and (4b) has no unstressed syllable between the first and second stressed syllables, one unstressed syllable between the second and third stressed syllables, and either zero or one syllable following the third stress, as depicted in the top left of Figure 8 (stressed 
syllables are indicated with accent marks [']). Likewise, phrases in (4c) and (4d) has comparable stress patterns as those in (4a) and (4b), except that there are two, not zero, unstressed syllables between the first and second stresses, as depicted in the top right of Figure 8.
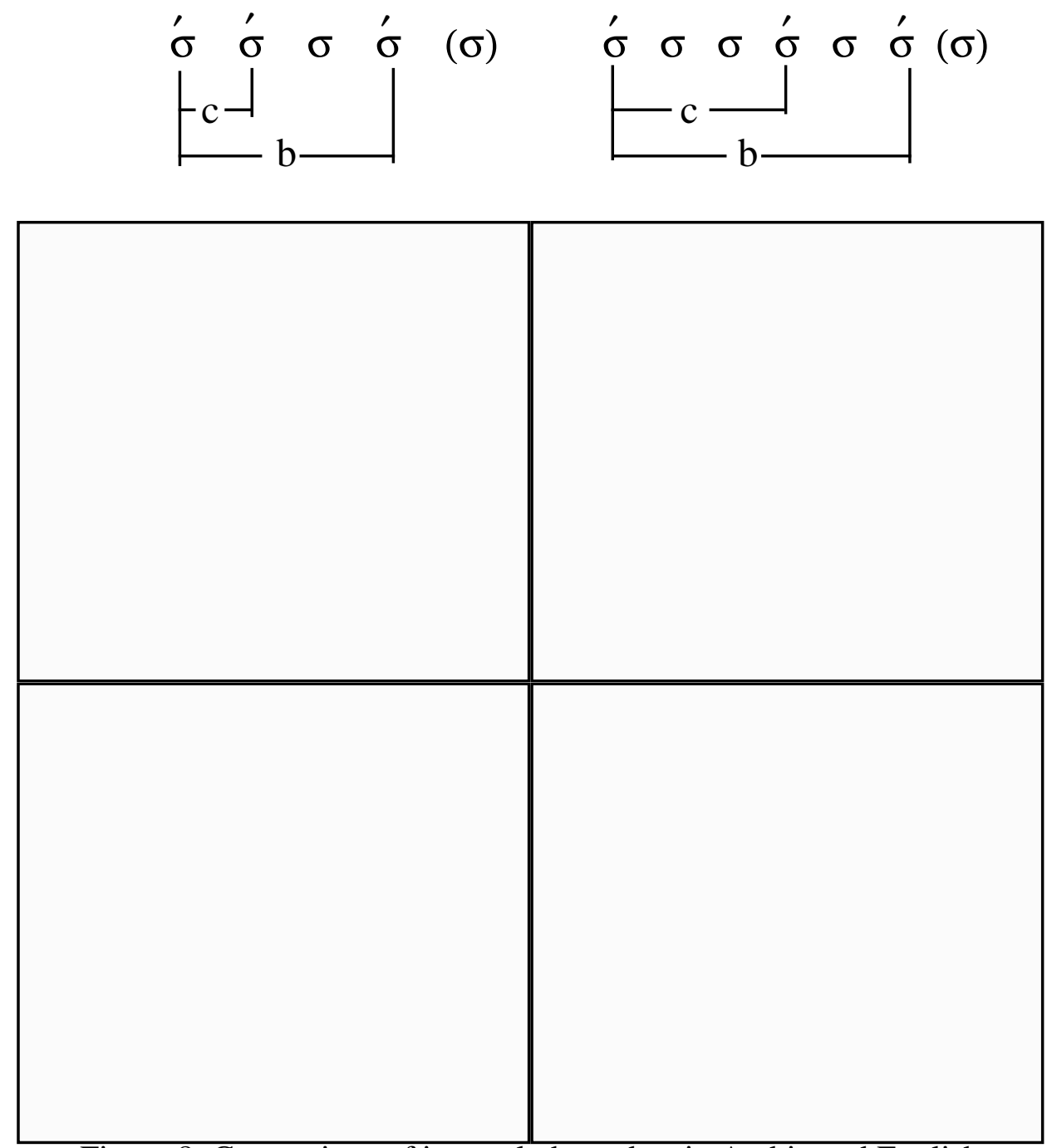

Figure 8: Comparison of internal phase data in Arabic and English

For each pair of phrases in (4), internal phase was calculated as the time of occurrence of the second stressed syllable relative to the interval between the first and third stressed syllables. This measure, shown as $c / b$ in Figure 8, captures the relative timing of the three stressed syllables in each phrase. Internal phase of 0.5 would indicate that the beats of the three stressed syllables occurred isochronously. By comparing internal phase measurements between Arabic and 
English phrases with the identical stress patterns, we can examine whether one language shows a stronger tendency toward stress isochrony than the other.

Results from the four pairs of phrases in (4) are shown in Figures $8(a)-8(d)$. Each panel shows mean internal phase (and standard deviation) of the second stressed syllable beat across all repetitions and speakers for one Arabic phrase, and compares them to results from an English phrase with the same stress pattern. For example, Figure 8(a) compares the mean internal phase of "baa-" in "mart baasim may" with the internal phase of "Doug" in "buy Doug a beer". The target syllable in both languages occurs earlier than internal phase 0.5 , but it occurs relatively closer to 0.5 in English than in Arabic. This indicates that English speakers more closely approximated acoustic isochrony of the three stressed syllables in the phrase than did Arabic speakers, who produced the first and second stressed syllables at a much shorter time interval than the second and third stressed syllables. Figure 8(b) shows the same result for phrases in (4b).

Figures $8(\mathrm{c})-8(\mathrm{~d})$ indicate that for phrases with two unstressed syllables between the first and second stressed syllables, internal phase of the second stress is later than 0.5. Phrases in these figures contain more unstressed syllables between the first and second stressed syllables than between the second and third stressed syllables. Crucially, mean internal phase of the target syllable is later for the Arabic phrases than for the English phrases, again suggesting that English speakers more closely approximate stress isochrony than do Arabic speakers.

\section{Discussion}

In this paper we reported the results of an experiment on Arabic rhythm in which we have used the speech cycling task. The task of repeating a text fragment along with periodic stimuli was found to be intuitively straightforward for the subjects. This is not surprising because similar behavior also occurs in natural settings, such as chanting, singing, public declarations, etc. Moreover, the speech cycling task has been used in other research on speech production and perception, such as the P-center studies.

In using the cycling task, we have used the notion of phase to measure timing. Specifically, we used external and internal phases to capture the relative timing of syllables. 
Our study on the rhythmic organization of Arabic shows similar results to previous studies on rhythmic organization. For instance, similar to the results of Cummins (1997) and Tajima (1998), we discovered that Arabic speakers find certain patterns more stable than others. In these stable patterns, simple harmonic phases such as $1 / 2$ seem to play a crucial role, as these phases are often just where stressed syllable beats are produced. Gradual changes in repetition rate was critical in revealing this tendency. Such behavior has been attested not only in speech, but also in other types of coordinated behavior (see Kelso, 1995 for a report on a finger wagging experiment for instance).

Moreover, our results support Tajima's (1998) proposal that languages differ with respect to the factors that contribute to the prominence of syllables. Tajima found evidence that phrase-final syllables are prominent in Japanese. In comparison, in English, stress syllables were found to be prominent. Our results indicate that Arabic is similar to English in that it is the stressed syllables that are prominent in the phrase. In both English and Arabic, the stressed syllables tended to occur at simple harmonic phases such as 1/2. Data from this study do not provide direct evidence that Japanese is "moratimed" per se, but the data do indicate that Japanese is not "stresstimed", unlike Arabic and English.

Finally, our results indicate that English speakers more closely approximate isochrony of stressed syllables within a phrase than Arabic speakers do. In other words, English is more strongly stresstimed than Arabic is. We believe this could be due to the degree of reduction of unstressed syllables. In English there is a greater amount of reduction in duration between stressed and unstressed syllables than in Arabic (see van Summers 1987; Zawaydeh and de Jong, 1999; de Jong and Zawaydeh, 1999). In addition to this, the Arabic and English stress systems differ in how they function in the language. In Arabic, stress is predictable by rule. In comparison, the stress location is variable in English. It depends on various morphological and lexical factors, and sometimes, stress is placed on some unpredictable locations in the word.

\section{Conclusion}

In this paper, a novel experimental method, speech cycling, was applied to Arabic and the results were compared to a previous parallel 
experiment that has been conducted by Tajima (1998). Analysis using external phase demonstrated that rhythmic modes in Arabic and English are similar to each other, while different from Japanese. Arabic and English speakers seem to pay close attention to the stressed syllables, producing them at simple harmonic phases. This result is consistent with the notion that these languages are "stress-timed". By contrast, Japanese speakers seem to show qualitatively different rhythmic preferences, placing the phrase-final syllable at simple harmonic phases. Despite their both being "stress-timed", Arabic and English also show systematic differences, according to internal phase measurements. Stressed syllables within a phrase deviated from a strictly isochronous sequence to a greater extent in Arabic than in English.

Overall, this study demonstrates that there are phonetically observable differences and similarities in rhythmic organization across languages. Some of these are captured by typological distinctions such as "stress-timed" vs. "syllable-timed". Conventional descriptions have held that these types of languages differ with respect to the units that occur at regular time intervals, i.e., stressed syllables vs. individual syllables (Abercrombie, 1976). Even though previous studies have generally failed to find acoustic evidence for these tendencies, data from Arabic, English, and Japanese suggest that there is something correct about the distinction, and that under suitably constrained conditions, Arabic and English behave differently from Japanese. However, other aspects of the data are not adequately captured by the conventional account. The finding that English appears to be "more stress-timed" than Arabic suggests that languages do not fall into discrete rhythmic categories, but rather show gradient variation in rhythmic tendencies.

\section{REFERENCES}

Abercrombie, D. (1967). Elements of General Phonetics. Chicago, IL: Aldine Publishing Company.

Beckman, M. (1986). Stress and Non-Stress Accent. Dordrecht: Foris.

Bolinger, D. (1965). Pitch accent and sentence rhythm. In Forms of English: Accent, Morpheme, Order . ed. by I. Abe \& T. Kanekiyo. Cambridge, MA: Harvard University press. 139-180.

Cummins, F. (1997). Rhythmic Coordination in English Speech: An Experimental Study. Ph.D. Dissertation, Indiana University.

Cummins, F. and Port, R. F. (1998). Rhythmic constraints on stress timing in English. Journal of Phonetics 26, 145-171. 
de Jong, K. (1994). The correlation of P-center adjustments with articulatory and acoustic events. Perception and Psychophysics 15, 447-460.

de Jong, K. (1999). Proportional and linear constancy in a repetitive speech production task. Journal of the Acoustical Society of Japan 106, 2243.

de Jong, K. and Zawaydeh, B. A. (1999). Stress, duration, and intonation in Arabic word-level prosody. Journal of Phonetics, 27, 3-22.

Fowler, C. (1983). Converging sources of evidence on spoken and perceived rhythms of speech: Cyclic production of vowels in monosyllabic stress feet. Journal of Experimental Psychology: General 112. 386-412.

Han, M. (1962). The feature of duration of Japanese. Onsei no Kenkyu (Study of Sounds) $10,65-75$.

Haraguchi, S. (1991). A Theory of Stress and Accent. Dordrecht: Foris.

Heliel, M. (1982). Stress timing in modern literary Arabic. Al-`Arabiya 15, 90106.

Hoequist, Jr. C. (1983). Syllable duration in stress, syllable, and mora-timed languages. Phonetica, 40, 203-237.

Hogg, R. and McCully, C. B. (1987). Metrical Phonology: A Coursebook. Cambridge, UK: Cambridge University Press.

Kelso, J. A. S. (1995). Dynamic Patterns: The Self-Organization of Brain and Behavior. Cambridge: MIT Press.

Marcus, S. M. (1981). Acoustic determinants of perceptual center (P-center) location. Perception \& Psychophysics 30, 247-256.

Miller, M. (1984). On the perception of rhythm. Journal of Phonetics 12, 75-83.

Morton, J., Martin, S. M., and Frankish, C. (1976). Perceptual centers (p-centers). Psychological Review, 83, 405-408.

O'Connor, J. D. (1965). The perception of time intervals. In Progress Report 2, 11-15. Phonetics Laboratory, University College London.

Pike, K. (1945). The Intonation of American English. Ann Arbor, MI: University of Michigan Press.

Port, R. F., Tajima, K., and Cummins, F. (1998). Speech and rhythmic behavior. In Non-linear Developmental Processes, Savelsburgh, G. and van der Maas, H. and van Geert, P. (eds.), Amsterdam: Elsevier, pp. 53-78.

Roach, P. (1982). On the distinction between 'stress-timed' and 'syllable-timed' languages. In Linguistic Controversies . ed. by D. Crystal. London: Edward Arnold. 73-79.

Scott, S. K. (1993). P-centers in Speech: An Acoustic Analysis. Ph.D. dissertation, University College London.

Shen, Y. and Peterson, G. G. (1982). Isochronism in English. University of Buffalo Studies in Linguistics, Occasional Papers, 9, 1-36.

Stark, R. (1986). Prespeech segmental feature development. In Language Acquisition, P. Fletcher and M. Garman (eds.), Cambridge, UK: Cambridge University Press, pp. 149-173.

Stetson, R. H. (1951). Motor Phonetics. Amsterdam: Elsevier.

Tajima, K. (1998). Speech Rhythm in English and Japanese: Experiments in Speech Cycling. Ph.D. dissertation, Indiana University.

Tajima, K. and Port, R. F. (to appear). Speech rhythm in English and Japanese. Papers in Laboratory Phonology 6, Cambridge: Cambridge University Press. 
Uldall, E. T. (1971). Isochronous stresses in R. P. In Form and Substance: Phonetic and Linguistic Papers Presented to Eli Fischer-Jorgensen. Ed by Hammerich, L. L., Jakobson, R., and Zwirner, E. Copehnagen: Akademisk Forlag. 205-210.

van Summers, W. (1987). Effects of stress and final-consonant voicing on vowel production: Articulatory and acoustic analyses. Journal of the Acoustical Society of America 82, 847- 863.

Williams, B. And Hiller, S. M. (1994). The question of randomness in English foot timing: A control experiment. Journal of Phoentics, 22, 423-429.

Zawaydeh, B. A. and de Jong, K. (1999). Stress, phonological focus, quantity, and voicing effects on vowel duration in Ammani Arabic. In Proceedings of the XIVth nternational Congress on Phonetic Sciences, San Francisco, pp. 451-454. 